\title{
Focusing of tandem bistatic SAR data using the chirp-scaling algorithm
}

\author{
Shichao Chen*, Mengdao Xing, Song Zhou, Lei Zhang and Zheng Bao
}

\begin{abstract}
Based on an exact analytical bistatic point target spectrum, an efficient chirp-scaling algorithm is proposed to correct the range cell migration of different range gates to the one of the reference range for tandem bistatic synthetic aperture radar data processing. The length of the baseline (baseline to range ratio) does not give a direct influence to the proposed algorithm, which can be applied to the processing of tandem bistatic data with a large baseline even when the baseline is equal to the range. No interpolation is needed during the entire processing, only fast Fourier transforms and phase multiplications are needed, which result in efficiency. The validity of the proposed algorithm has been verified by simulated experiments.
\end{abstract}

Keywords: Chirp-scaling algorithm, Tandem bistatic configuration, Point target (PT) spectrum

\section{Introduction}

Due to the wide applications of bistatic synthetic aperture radar (SAR), much attention has been paid to it [1-3]. As one of the translational invariant (TI) configurations, the tandem configuration, in which, the transmitter and receiver move along the same track, has a relatively simple formation structure, and can easily be accomplished in engineering. The real bistatic system (TanDEM-X) of this configuration has already obtained well-focused bistatic images and interferograms [3].

As is known, besides the time, frequency, and antenna pointing synchronic problems, due to the separation of the transmitter and the receiver [4,5], fast imaging algorithms are also in search. Although the time domain method is the best in theory, it requires huge computation [6]. Rodriguez-Cassola et al. [7] proposed an efficient time domain imaging algorithm that has small computational burden respect to the direct backprojection (DBP) algorithm and high accuracy for the general time-varying configuration. The precise point target (PT) spectrum of bistatic SAR is the precondition of fast imaging algorithms in the frequency domain. However, we cannot acquire accurate bistatic PT spectrum through the principle of stationary phase (POSP) because of the double-square-root term existed

\footnotetext{
* Correspondence: chenshichao725@gmail.com

National Lab of Radar Signal Processing, Xidian University, Xi'an 710071, China
}

in the range history of bistatic SAR. Many effective spectrums have been proposed to handle the problem [8-18], such as the dip move out [8,9], Lofffeld's bistatic formula [10-12], and method of series reversion (MSR)-based spectrums [13-17]. The latter two are suitable for any general bistatic configuration, and have wider application areas. A novel frequency domain PT spectrum able to address the general bistatic configuration accurately has also recently been proposed [18]. However, these spectrums are all approximate ones under certain conditions. Focusing on the well-known tandem configuration, based on the geometry-based bistatic formula (GBF) method [19], Wu et al. [20,21] deduced an exact analytical PT spectrum, and proves that all the spectrums aforementioned are approximations of the very spectrum under certain conditions. However, "stolt" interpolation process with computational burden is inevitable by using the range migration algorithm imaging algorithm [20].

Due to the effectiveness and efficiency of the chirpscaling algorithm [22,23], a chirp-scaling algorithm is proposed based on this very exact analytical spectrum for tandem configuration in this article, which corrects the range cell migration (RCM) through phase multiplication with efficiency. Just as the monostatic case, no interpolation operation is performed during the whole process, which improves the effectiveness of the imaging process and also guarantees the focusing quality. It is capable of dealing with bistatic SAR data in tandem 
configuration without the influence of the length of the baseline, even in the extreme situation when the baseline is equal to the range (baseline to range ratio equals 1 ). The correctness of each step can be tested and guaranteed by the comparison with the monostatic case.

The remainder of the article is organized as follows. The geometry of the bistatic SAR configuration that is being discussed is introduced in Section 2. And then, the proposed chirp-scaling algorithm is discussed in detail in Section 3. Simulated experiments are carried out in Section 4 to validate the proposed algorithm. In Section 5 , we draw conclusions.

\section{Geometry of tandem bistatic SAR}

Figure 1 shows the geometry relationship of the bistatic SAR of equal velocity vectors in tandem configuration. The transmitter $T x$ and the receiver $R x$ move along the same track with the same speed vector $v_{0}, \theta_{T}$ and $\theta_{R}$ are the instantaneous angles from the target to the platforms, $R_{T}$ and $R_{R}$ are the corresponding slant ranges, $R_{B}$ is the closest distance from the target to the flight path, $\beta$ is the half bistatic angle, and $h_{x}$ is half the length of the baseline.

Making use of the tangent of the half bistatic angel, the exact analytical expression of the half bistatic angle can be obtained based on the GBF method in this configuration $[20,21]$.

$$
\begin{gathered}
\beta=\arctan \left(\frac{-M}{2}+\frac{P}{2}\right) \\
P=\sqrt{-M^{2}-4+8 K_{R}^{2} R_{B} /\left(h_{x} K_{X}^{2} M\right)}, M=\sqrt{2 y-2} \\
y=\left[-q / 2+\sqrt{(q / 2)^{2}+(p / 3)^{3}}\right]^{1 / 3}+\left[-q / 2-\sqrt{(q / 2)^{2}+(p / 3)^{3}}\right]^{1 / 3}+1 / 3 \\
p=-\left(\frac{4}{3}-\frac{4 K_{R}^{2}}{K_{X}^{2}}\right), q=\frac{2}{3}\left(1-\frac{4 K_{R}^{2}}{K_{X}^{2}}\right)-\frac{2}{27}-\frac{2 K_{R}^{4} R_{B}^{2}}{h_{x}^{2} K_{X}^{4}}
\end{gathered}
$$

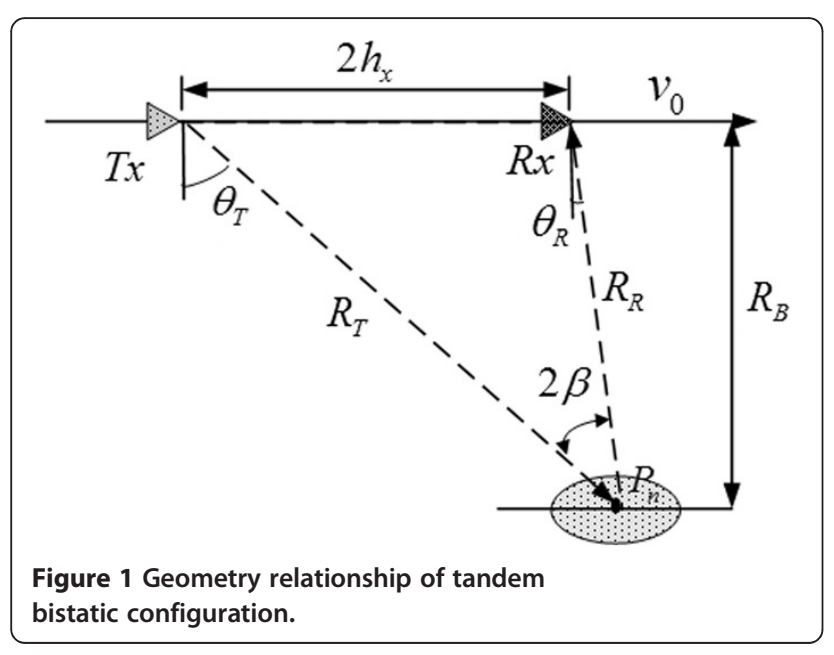

where $K_{R}$ denotes the range wavenumber, and $K_{X}$ denotes the azimuth wavenumber.

\section{Chirp-scaling algorithm for tandem bistatic SAR}

Using the GBF method [19], the two-dimensional (2D) expression of the PT spectrum in tandem configuration with identical velocity vectors for both the transmitter and the receiver in the wavenumber domain can be written as

$$
\begin{gathered}
S\left(\Delta K_{R}, K_{X}\right)=W_{K_{R}} W_{K_{X}} \exp \left[-j \frac{\left(K_{R}-K_{R c}\right)^{2}}{2 b}\right] \exp \left(-j K_{X} x_{n}\right) \\
\exp \left[-j\left(R_{B}+h_{x} \tan \beta\right) \sqrt{4 K_{R}^{2} \cos ^{2} \beta-K_{X}^{2}}\right]
\end{gathered}
$$

where $W_{K_{R}}=\operatorname{rect}\left[\frac{K_{R}-K_{R}}{b c T_{p}}\right]$ represents the spectral shape of the transmitted pulse, rect[ . ] is the rectangular window function, $K_{R c}=2 \pi f_{c} / c, f_{c}$ is the carrier frequency, $c$ is the speed of light, $\Delta K_{R}=K_{R}-K_{R c}=2 \pi f_{r} / c$, and $f_{r}$ is the range frequency, $T_{p}$ is the pulse duration, $W_{K_{X}}$ is the shape of the azimuth wavenumber spectrum, $1 / b=2 \pi \gamma / c^{2}$, and $\gamma$ is the range chirp rate, $x_{n}$ is the azimuth position at where the imaged target is located.

Inspecting (3), it becomes obvious that the expression reduces to the very one of the monostatic case when $\beta=$ $0, h_{x}=0$. There are no approximations during the whole deduction of $\beta$ expressed by Equation (1). It is an exact analytical one, so is the 2D spectrum expressed by Equation (3). Based on Equation (3), one can deduce a series of imaging algorithms as the monostatic case. We focus on the precise and efficient chirp-scaling algorithm in this article.

Chirp-scaling algorithm employs the property of the transmitted signal, whose range frequency is linear variant. A scaling function is multiplied with the signal to eliminate the space variance of the RCM curves of the targets in different range gates in range-Doppler domain [22]. To get the formation of the scaling function, one needs to know the range migration trajectory and the frequency modulation (FM) rate of the signal.

Like the monostatic case, a Taylor series expansion at $K_{R}=K_{R c}$ up to the second term results in

$$
\begin{aligned}
S\left(\Delta K_{R}, K_{X}\right)= & W_{k_{R}} W_{k_{X}} \exp \left(j \phi_{0}-j \phi_{1} \Delta K_{R}+j \phi_{2} \Delta K_{R}^{2}\right) \\
& \exp \left(-\frac{\Delta K_{R}^{2}}{2 b}\right) \exp \left(-j K_{X} x_{n}\right)
\end{aligned}
$$

where $\phi_{0}$ is the azimuth focusing function, $\phi_{1}$ is the range migration factor for tandem bistatic SAR, $\phi_{2}$ is the secondary range compression term, and the detailed expressions of these three parameters are given below. The last exponential term in (4) indicates the azimuth position at where the imaged target is located. Note that, 
the orders of Taylor series expansion can be adjusted to meet the accuracy of satisfactory imaging results. For the situation with small squint angles, up to the quadratic term is quite enough. The signal has been converted into the baseband during the process. In Equation (4)

$$
\begin{aligned}
\phi_{0}= & -\left(R_{B}+h_{x} \tan \beta_{0}\right) \cdot M_{1} \\
\phi_{1}= & \left(h_{x} \beta_{0}{ }^{\prime} \sec ^{2} \beta_{0}\right) \cdot M_{1} \\
& +\left(R_{B}+h_{x} \tan \beta_{0}\right) \cdot M_{2} / M_{1} \\
\phi_{2}= & -\frac{M_{4} \cdot M_{1}}{2}-\left(h_{x} \beta_{0}{ }^{\prime} \sec ^{2} \beta_{0}\right) \frac{M_{2}}{M_{1}} \\
& -R_{B}\left(1+\rho \tan \beta_{0}\right) \frac{M_{3}}{2 M_{1}} \\
& +R_{B}\left(1+\rho \tan \beta_{0}\right) \frac{M_{2}^{2}}{2 M_{1}^{3}},
\end{aligned}
$$

where $\rho=h_{x} / R_{B}$ in Equation (7) is the baseline to range ratio. In the equations above

$$
\begin{aligned}
M_{1} & =\sqrt{4 K_{R c}^{2} \cos ^{2} \beta_{0}-K_{X}^{2}} \\
M_{2} & =4 K_{R c} \cos ^{2} \beta_{0}-2 K_{R c}^{2} \beta_{0}{ }^{\prime} \sin \left(2 \beta_{0}\right) \\
M_{3} & \left.=4 \cos ^{2} \beta_{0}-8 K_{R c} \beta_{0}{ }^{\prime} \sin \left(2 \beta_{0}\right)-2 K_{R c}^{2} \beta_{0}^{\prime}\right\} \sin \left(2 \beta_{0}\right)(8) \\
& -4 K_{R c}^{2}\left(\beta_{0}{ }^{\prime}\right)^{2} \cos \left(2 \beta_{0}\right) \\
M_{4} & =h_{x} \beta_{0}^{\prime}{ }^{\prime} \sec ^{2} \beta_{0}+2 h_{x}\left(\beta_{0}{ }^{\prime}\right)^{2} \tan \beta_{0} \sec ^{2} \beta_{0} \\
\beta_{0} & =\left.\beta\right|_{K_{R}=K_{R c}, \beta_{0}^{\prime}}=\left.\frac{\partial \beta}{\partial K_{R}}\right|_{K_{R}=K_{R c}} \beta_{0}^{\prime \prime}=\left.\frac{\partial^{2} \beta}{\partial K_{R}^{2}}\right|_{K_{R}=K_{R c}}
\end{aligned}
$$

Note that, $\phi_{2}$ is range dependent, but in the situation when bistatic SAR works with small squint angles, the range dependence can be neglected. We can consider the introduction of the nonlinear chirp-scaling algorithm (NCSA) [23] when the range dependence has to be taken into consideration. In the following step, we deduce the chirp-scaling imaging algorithm for tandem bistatic SAR with small squint angles.

In addition, the formations of these parameters shown by Equations (5)-(7) seem rather complex because of the complex formation of the exact analytical spectrum that we are based on. The exact expression of the half bistatic angle is obtained through the solution of a four-order equation, which results in a relatively complex formation of the solution of the exact analytical spectrum. Fortunately, the correctness of these parameters can be tested by comparing them with the monostatic ones when $\beta=0, h_{x}=0$. They will turn out to be $\phi_{0}=$ $-R_{B} \sqrt{4 K_{R c}^{2}-K_{X}^{2}}, \phi_{1}=2 R_{B} / \sqrt{1-\frac{K_{X}^{2}}{4 K_{R c}^{2}}}, \phi_{2}=\frac{2 R_{B} K_{X}^{2}}{\left(\sqrt{4 K_{R c}^{2}-K_{X}^{2}}\right)^{3}}$. They are all in accordance with the monostatic ones.

As can be seen from $\phi_{1}$ expressed by (6), which is not only dependent on the Doppler frequency $K_{X}$, but also changes with range. That is to say, the difference of the
RCM curves in different range gates exists. We expand $\phi_{1}$ at $R_{s}$ (the closest distance to the flight track from the scene center) to its Taylor series and reserve the linear term, which takes the form

$$
\phi_{1}=A_{\text {ref }}+\Delta r / B_{\text {ref }}
$$

where

$$
\begin{aligned}
A_{\mathrm{ref}}=\left.\phi_{1}\right|_{R_{B}=R_{s}}= & {\left.\left[h_{x} \beta_{0}^{\prime} \sec \left(\beta_{0}\right)^{2} M_{1}+\left(R_{B}+h_{x} \tan \beta_{0}\right) \frac{M_{2}}{M_{1}}\right]\right|_{R_{B}=R_{s}} } \\
\frac{1}{B_{\mathrm{ref}}}=\left.\frac{\partial \phi_{1}}{\partial R_{B}}\right|_{R_{B}=R_{s}}= & {\left[\left(h_{x} \sec ^{2} \beta_{0} \frac{\partial \beta_{0}^{\prime}}{\partial R_{B}}+2 h_{x} \beta_{0}^{\prime} \sec ^{2} \beta_{0} \tan \beta_{0} \frac{\partial \beta_{0}}{\partial R_{B}}\right) M_{1}\right.} \\
& +h_{x} \beta_{0}^{\prime} \sec ^{2} \beta_{0} \frac{\partial M_{1}}{\partial R_{B}}+\left(1+h_{x} \sec ^{2} \beta_{0} \frac{\partial \beta_{0}}{\partial R_{B}}\right) \frac{M_{2}}{M_{1}} \\
& \left.+\left(R_{B}+h_{x} \tan \beta_{0}\right)\left(\frac{1}{M_{1}} \frac{\partial M_{2}}{\partial R_{B}}-\frac{M_{2}}{M_{1}^{2}} \frac{\partial M_{1}}{\partial R_{B}}\right)\right] \mid R_{B}=R_{S}
\end{aligned}
$$

$\Delta r=2 R_{B}-2 R_{s}$ represents the range position respect to the scene center. The error caused by the approximation of (10) is far less than the range resolution, whose influence can be neglected under the simulation parameters of this article given in the following part. Note that, when the amplitude of this approximation is large enough, we can divide the data into blocks in the range direction to eliminate the influence of this approximation. As can be seen, $A_{\text {ref }}$ and $B_{\text {ref }}$ are both determined by the parameters of the reference range. To see them clearly, we also take a look at them in monostatic case. When $\beta_{0}=0, h_{x}=0$, we have

$$
\begin{aligned}
& A_{\text {ref }}=2 R_{s} / \sqrt{1-\frac{K_{X}^{2}}{4 K_{R c}^{2}}} \\
& \frac{1}{B_{\text {ref }}}=2 / \sqrt{1-\frac{K_{X}^{2}}{4 K_{R c}^{2}}}
\end{aligned}
$$

We can see that the familiar term $\sqrt{1-K_{X}^{2} /\left(4 K_{R c}^{2}\right)}$ existed in the denominator of both equations is the cosine of the squint angle in the view of geometry just as the monostatic case. Equation (13) is just in accordance with the migration trajectory of the target in the reference range, and we have

$$
A_{\text {ref }}+\Delta r / B_{\text {ref }}=2 R_{B} / \sqrt{1-\frac{K_{X}^{2}}{4 K_{R c}^{2}}}
$$

As can be seen, the result is identical to the expression of the RCM term in monostatic case (i.e., tandem bistatic configuration is just a special case of monostatic case). The term $2 R_{B}$ in the numerator of the equation is 
in accordance with the sum of the range history of the transmitter and the receiver, which degenerates into $R_{B}$ in monostatic case. Substituting (10) into (4), the signal arrives at

$$
\begin{gathered}
S\left(\Delta K_{R}, K_{X}\right)=W_{k_{R}} W_{k_{X}} \exp \left(j \phi_{0}\right) \exp \left(-j K_{X} x_{n}\right) \\
\exp \left[-j\left(A+\Delta r / B_{\mathrm{ref}}\right) \Delta K_{R}\right] \exp \left[j \frac{\Delta K_{R}^{2}}{2 b_{m}}\right]
\end{gathered}
$$

where $\frac{1}{2 b_{m}}$ is the FM rate of the signal in the 2D wavenumber domain.

$$
\frac{1}{2 b_{m}}=-\frac{1}{2 b}+\frac{1}{2 b_{S R C}} \Rightarrow b_{m}=\frac{b b_{S R C}}{b-b_{S R C}}
$$

where $b_{S R C}=\frac{1}{2 \phi_{2}}$. An inverse Fourier transform (IFT) is performed in range to transform the signal into the $\left(y_{s}\right.$, $K_{X}$ ) domain.

$$
\begin{gathered}
S\left(y_{s}, K_{X}\right)=W_{r} W_{k_{X}} \exp \left(j \phi_{0}\right) \exp \left(-j K_{X} x_{n}\right) \\
\exp \left[-j b_{m}\left(y_{s}-A_{\text {ref }}-\Delta r / B_{\text {ref }}\right)^{2} / 2\right]
\end{gathered}
$$

where $W_{r}=\operatorname{rect}\left[\frac{b_{m}\left(y_{s}-A_{\text {ref }}-\Delta r / B_{\text {ref }}\right)}{b c T_{p}}\right], y_{s}$ represents the range position relative to the imaging scene center, $A_{\text {ref }}$ $+\Delta r / B_{\text {ref }}$ represents the RCM term. We can tell that $B_{\text {ref }}$ $\left(K_{X}\right)$ is Doppler wavenumber dependent, which makes $\Delta r / B_{\text {ref }}$ different in different range gates. Making use of the characteristic of the linear frequency modulated signal to eliminate the different RCM, which is dependent on range. The signal is multiplied by a scaling function to equalize the RCM of all ranges to the one of the scene center. Resembling monostatic case [22], the scaling function is constructed as

$$
H_{\text {scale }}\left(y_{s}, K_{X}\right)=\exp \left[-j b_{m} D\left(K_{X}\right)\left(y_{s}-A_{\text {ref }}\right)^{2} / 2\right]
$$

where

$$
D\left(K_{X}\right)=\frac{1}{B_{\text {ref }}}-1
$$

As can be seen again, $D\left(K_{X}\right)$ is the familiar scaling factor as the chirp-scaling algorithm in monostatic case. Transforming the signal into the $2 \mathrm{D}$ wavenumber domain after (19) is multiplied by (18). The signal moves to

$S\left(\Delta K_{R}, K_{X}\right)=W_{k_{X}} W_{k_{R}} \exp \left(j \phi_{0}\right) \exp \left(-j K_{X} x_{n}\right) \exp \left[j B_{\text {ref }} \Delta K_{R}^{2} /\left(2 b_{m}\right)\right]$ $\exp \left(-j \Delta K_{R} \Delta r\right) \exp \left(-j \Delta K_{R} A_{\text {ref }}\right) \exp \left\{-j b_{m} D\left(K_{X}\right)\left[1+D\left(K_{X}\right)\right] \Delta^{2} r / 2\right\}$

We take a look at the exponential terms of Equation (21): the first one is the term for azimuth-matched filtering; the second one indicates the azimuth position of the imaged target as aforementioned; the third one is the range compression term, which is range dependent; the fourth one indicates the position where the target is located at in the range direction; the fifth one is the bulk RCM term for targets of all ranges; the last one is the residual phase error term, which turns out to be the familiar one of monostatic case when $\beta=0, h_{x}=0$.

From (21), we can see that the variation of the RCM in different range gates has been eliminated after the scaling operation. The RCM curves of the targets in different range gates have been equalized to the one of the reference ranges. The linear phase term $\Delta K_{R} \Delta r$ which indicates the range position of the targets has emerged as expected.

As to the range compression, it is range dependent since $b_{m}$ is range dependent. However, the range dependence of the SRC is not significant in situation when the bistatic SAR system works with small squint angles. We can compensate it by using the parameters of the reference ranges. The range compression is carried out by

$$
H_{\mathrm{SRC}}\left(\Delta K_{R}, K_{X}\right)=\exp \left[-j B_{\mathrm{ref}} \Delta K_{R}^{2} /\left(2 b_{m 0}\right)\right]
$$

where $\quad b_{m 0}=\frac{b b_{S R C 0}}{b-b_{S R C 0}}, \quad b_{S R C 0}=\frac{1}{2 \phi_{20}}, \quad \phi_{20}=\left.\phi_{2}\right|_{R_{B}=R_{s}}$. The error of this approximation can be expressed as $\phi_{\mathrm{Err}}=$ $\frac{B_{\mathrm{re}} \Delta K_{R}^{2}}{2 b_{m}}-\frac{B_{\mathrm{ret}} \Delta K_{R}^{2}}{2 b_{m 0}}$. The amplitude of $\left|\phi_{\mathrm{Err}}\right|$ in the cases of low squint angle is less than $\pi / 4$, the influence introduced by the approximation can be neglected, and ideal focusing results can be obtained. However, when the system works with high squint angles, the amplitude of $\left|\phi_{\mathrm{Err}}\right|$ will be larger than $\pi / 4$, if the range compression is still performed by (22), poor focusing result will emerge. And in the situation of high squint angles, NCSA in monostatic case can be introduced to handle the problem [23]. In practice, we can calculate the amplitude of $\left|\phi_{\mathrm{Err}}\right|$, if the amplitude is less $\pi / 4$, we can carry out the proposed algorithm. Besides, as can be seen, the further the distance from the target to the scene center in range, the bigger the error will be. Thus when the imaging swath is large enough that the error will also be larger than $\pi / 4$, we can divide the data into range blocks to handle the problem as is discussed for the monostatic case [23].

Then, according to Equation (21), the bulk RCM correction function is subsequently performed

$$
H_{\mathrm{RMC}}\left(\Delta K_{R}, K_{X}\right)=\exp \left(j \Delta K_{R} A_{\text {ref }}\right)
$$

The residual phase compensation is implemented after a fast Fourier transform (FFT) in range. The signal is again transformed into the $\left(y_{s}, K_{X}\right)$ domain. 
Table 1 Parameters of tandem bistatic SAR in two cases with different baselines

\begin{tabular}{|c|c|c|c|c|}
\hline & Case I & & Case II & \\
\hline Range to reference target & $20,500 \mathrm{~m}(\mathrm{~T})$ & $20,304 \mathrm{~m}(\mathrm{R})$ & $22,589 \mathrm{~m}(\mathrm{~T})$ & $22,142 \mathrm{~m}(\mathrm{R})$ \\
\hline Squint angle & $12.7^{\circ}(\mathrm{T})$ & $-9.93^{\circ}(\mathrm{R})$ & $27.7^{\circ}(\mathrm{T})$ & $-25.4^{\circ}(\mathrm{R})$ \\
\hline Length of the baseline & & & & \\
\hline Doppler bandwidth & & & & \\
\hline PRF & & & & \\
\hline Range bandwidth & & & & \\
\hline Range chirp bandwidth & & & & \\
\hline Carrier frequency & & & & \\
\hline Platform velocity & & & & \\
\hline Closest range to the flight track & & & & \\
\hline
\end{tabular}

$$
H_{\mathrm{RES}}\left(y_{s}, K_{X}\right)=\exp \left\{j b_{m} D\left(K_{X}\right)\left[1+D\left(K_{X}\right)\right] \Delta^{2} r / 2\right\}
$$

The azimuth compression is performed in the following, and the azimuth-matched filter is given as

$$
H_{A Z I}\left(y_{s}, K_{X}\right)=\exp \left[j\left(R_{B}+h_{x} \tan \beta_{0}\right) \cdot M_{1}\right]
$$

Note that, $R_{B}, \beta_{0}$, and $M_{1}$ are all range-dependent parameters, we have to implement this step in each range gate. At the end of the whole imaging algorithm, an IFT is performed to transform the focused data into the complex image domain.

\section{Simulations}

Table 1 ( $\mathrm{T}$ in the brackets represents the transmitter and $\mathrm{R}$ represents the receiver) illustrates the parameters of bistatic SAR in tandem configuration with equal velocity vectors in two cases with different baselines (baseline to range ratio). Since the tandem configuration discussed here is a TI case, we put seven targets in the imaging scene with the same azimuth position, whose coordinates are T1 $(0,-1500), \mathrm{T} 2(0,-1000), \mathrm{T} 3(0,-500), \mathrm{T} 4$ $(0,0)$, T5 $(0,500)$, T6 $(0,1000)$, and T7 $(0,1500)$, respectively. First, we come to case $\mathrm{I}$, the result of range compression is shown in Figure 2a (T4-T7 are taken for illustration), we can see squinted versions of the RCM due to the squint angle of the transmitter and the receiver, and the result of the RCM correction obtained by using the proposed chirp-scaling algorithm is shown in Figure 2b. As can be seen, the RCM of the targets is removed properly.

All the seven targets are well focused by using the proposed chirp-scaling algorithm. To determine the performance of the proposed algorithm, we compare it with the chirp-scaling algorithm based on the MSR spectrum. The imaging results of the reference range target $\mathrm{T} 4$ and the far range target T7 are taken for illustration for this case. The final contour plot of T4 is shown in Figure 3a.
The range impulse response is shown in Figure 3b, while the azimuth impulse response is shown in Figure 3c. The corresponding results obtained by using the chirpscaling algorithm based on the MSR spectrum are shown in Figure $3 \mathrm{~d}-\mathrm{f}$ in accordance. The imaging results of the furthest range target T7 by using the two algorithms are shown in Figure 4 with the same sequence as Figure 3. The values of the impulse response width (IRW), the peak sidelobe ratio (PSLR), and the integrated sidelobe ratio (ISLR) of T4 and T7 obtained by using the proposed algorithm are provided in Table 2, and the corresponding values by using the MSR spectrum-based algorithm are provided in Table 3. Rectangular weighting is used for both the range and the azimuth processing. As can be seen, they all agree with the theoretical values. The imaging results under the two different spectrums are almost the same in this case with the length of the baseline $8000 \mathrm{~m}$ (baseline to range ratio 0.4). Ideal focusing can be obtained by using both the algorithms.

In the following, we come to Case II with the baseline $20,000 \mathrm{~m}$, which is equal to the range (baseline to range ratio 1 ). We take targets $\mathrm{T} 4, \mathrm{~T} 5, \mathrm{~T} 6$, and $\mathrm{T} 7$ for illustration in this case. The imaging results of $\mathrm{T} 4$ by using the

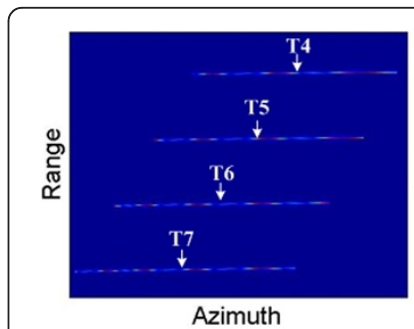

(a)

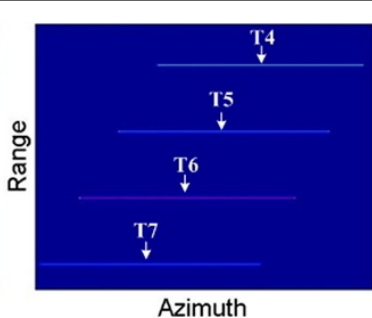

(b)
Figure 2 Trajectories of the targets before and after the RCM correction. (a) Trajectories of the targets after range compression. (b) Trajectories of the targets after the RCM correction. 


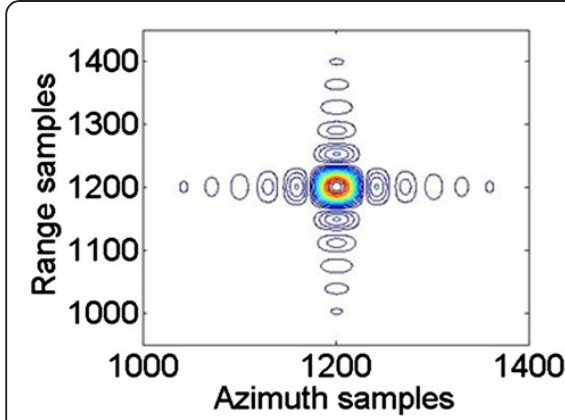

(a)

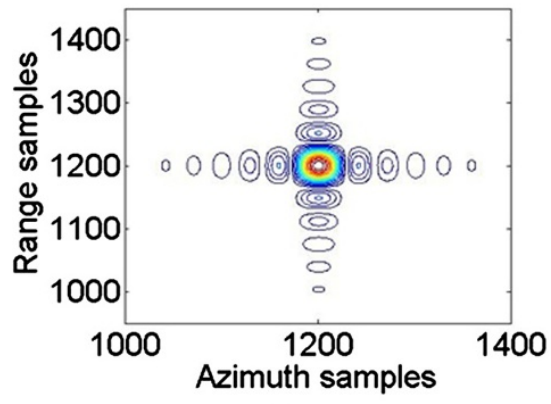

(d)

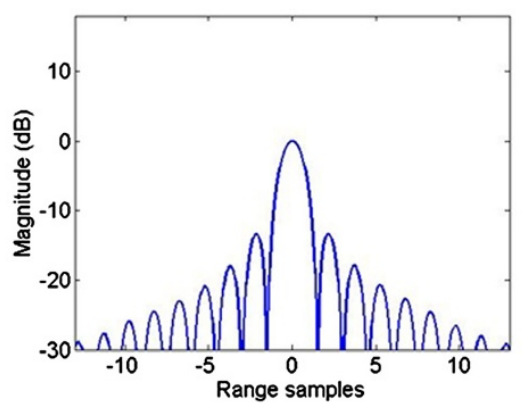

(b)

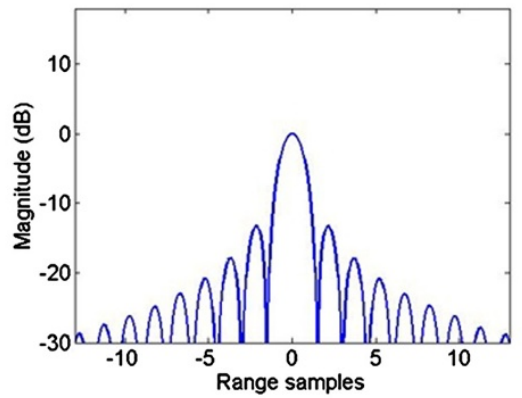

(e)

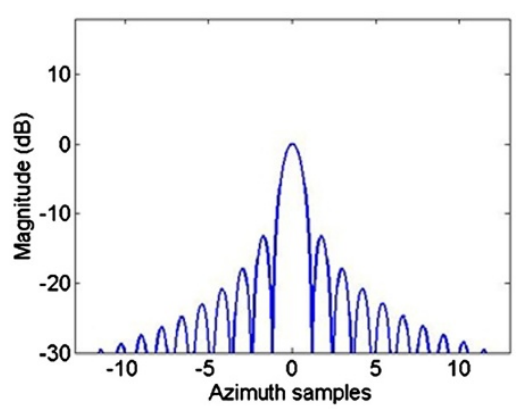

(c)

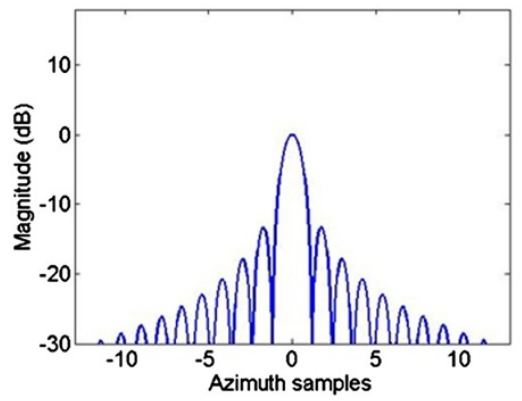

(f)

Figure 3 Imaging results of target T4 for case I: (a) contour plots obtained by using the proposed chirp-scaling algorithm; (b) range impulse response obtained by using the proposed chirp-scaling algorithm; (c) azimuth impulse response obtained by using the proposed chirp-scaling algorithm; (d) contour plots obtained by using the MSR spectrum-based algorithm; (e) range impulse response obtained by using the MSR spectrum-based algorithm; (f) azimuth impulse response by using the MSR spectrum-based algorithm.

two algorithms based on different spectrums are shown in Figure 5, while corresponding results for $\mathrm{T} 5$ are shown in Figure 6, T6 in Figure 7, and T7 in Figure 8 for this case. The detailed values of the IRW, PSLR, and ISLR under the proposed algorithms are given in Table 4, and the corresponding ones for the MSR-based algorithm are illustrated in Table 5 . As can be seen, the imaging results in the range direction of all the four targets are good enough just as in Case I. However, the focusing quality in the azimuth direction differs much under different algorithms with different range distances from the position of the target to the scene center.

From the imaging results, we can tell that satisfactory imaging results can be obtained by using both the algorithms for the reference target T4 as in Case I. And all the other three targets are also well focused by using the proposed chirp-scaling algorithm. Thanks to the high precision of the spectrum that we based on, the proposed algorithm is not sensitive to the baseline (baseline to range ratio), and its performance is satisfying in both cases with different baselines, even in this case when the baseline is equal to the range. And from the comparison of the focusing quality in the azimuth direction of T5, as shown in Figure 6c,f, we can tell that the focusing quality of the MSR spectrum-based algorithm is not as good as that obtained based on the exact analytical one. And for T6, the azimuth focusing quality based on the MSR spectrum is much worse than the proposed one, as shown in Figure 7c,f. From the descriptions of Table 5, we can tell that the focusing quality for T6 is not as good as that obtained by using the proposed algorithm, and the performance for T7 by the MSR-based algorithm is even worse, as shown Figure 8f. From the imaging results, we can see that ideal focusing quality can be obtained in range by using both the algorithms, while the focusing quality differs much in azimuth. Since both algorithms can realize RCMC with satisfactory results, and the SRC term has been compensate appropriately, hence ideal focusing quality can be obtained in the range direction under both the algorithms. As to the azimuth direction, the focusing quality is based on the precision of the spectrum that the imaging algorithm is based on. Since the MSR spectrum is not as accurate as the exact analytical one, the proposed algorithm outperforms the other one with large baselines.

From all these results, we can tell that with the increasing of the distance from the position of the target to the scene center in the range direction, the focusing 


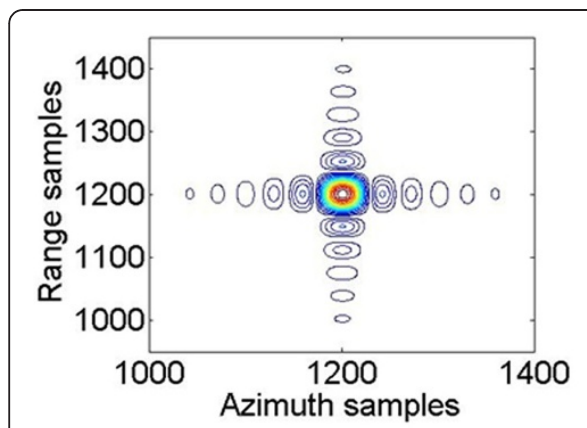

(a)

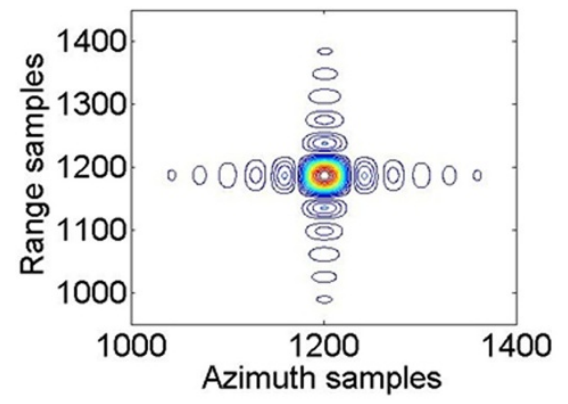

(d)

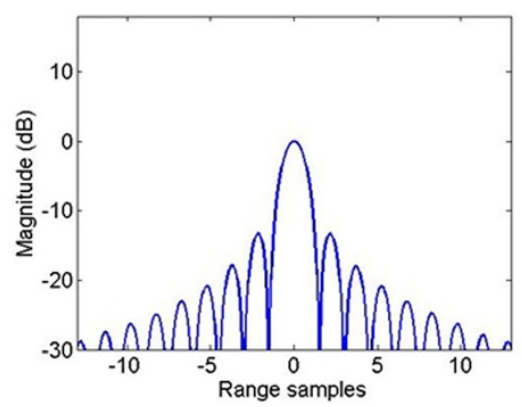

(b)

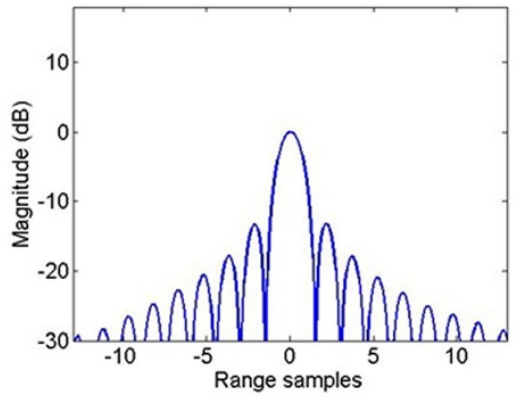

(e)

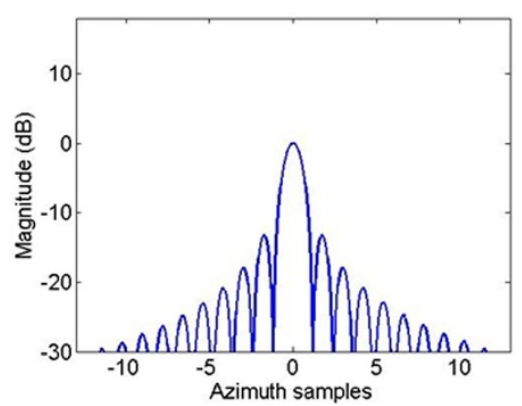

(c)

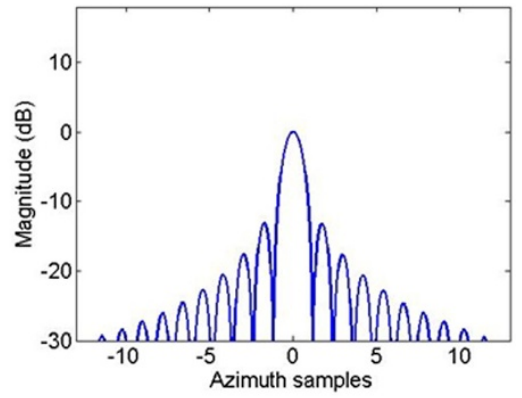

(f)

Figure 4 Imaging results of target T7 for case I: (a) contour plots obtained by using the proposed chirp-scaling algorithm; (b) range impulse response obtained by using the proposed chirp-scaling algorithm; (c) azimuth impulse response obtained by using the proposed chirp-scaling algorithm; (d) contour plots obtained by using the MSR spectrum-based algorithm; (e) range impulse response obtained by using the MSR spectrum-based algorithm; ( $f$ ) azimuth impulse response by using the MSR spectrum-based algorithm.

quality degrades dramatically by using the MSR-based chirp-scaling algorithm, while satisfying results can be obtained by using the proposed one. The reason for this phenomenon lies in the fact that the proposed chirpscaling algorithm is based on an exact analytical bistatic PT spectrum, while the one based on the MSR spectrum is an approximate one. Although the MSR spectrum is a spectrum with high precision, it is still an approximation to some extent.

Note that, as is known, the precision of the MSR spectrum is controllable by the orders of Taylor series expansion. Here, we only expand it to the third-order. To get better focusing results, we can expand the spectrum to higher orders. However, here the MSR spectrum-based algorithm is just used for comparison to

Table 2 Quality parameters of impulse-response function for targets T4 and T7 in Case I by using the proposed algorithm

\begin{tabular}{lccccccc}
\hline & \multicolumn{3}{c}{ Range } & & \multicolumn{3}{c}{ Azimuth } \\
\cline { 2 - 3 } & IRW(cells) & PSLR(dB) & ISLR(dB) & IRW(cells) & PSLR(dB) & ISLR(dB) \\
\hline $\mathrm{T} 4$ & 1.5000 & -13.2802 & -9.9266 & & 1.1875 & -13.2857 & -9.7707 \\
\hline $\mathrm{T} 7$ & 1.5000 & -13.2667 & -9.9055 & & 1.1875 & -13.2494 & -9.7228 \\
\hline
\end{tabular}

validate the effectiveness of the proposed one, so we only expand it to the third-order for simplicity.

And to further prove the effectiveness of the proposed algorithm for tandem configuration. We compare it with another two advanced imaging algorithms for bistatic SAR. One of them is the bistatic fast factorized backprojection (BFFBP) algorithm carried out in the time domain with small computational burden and high accuracy [7], and the other one is the chirp-scaling imaging algorithm based on the 2D POSP [18]. Both the algorithms are capable of handling bistatic SAR data in a more flexible configuration, and thus can be applied into wider application areas.

Focusing on the tandem bistatic configuration with identical velocity vector for the transmitter and the

Table 3 Quality parameters of impulse-response function for targets T4 and T7 in Case I by using the MSR-based algorithm

\begin{tabular}{lcccccccc}
\hline & \multicolumn{3}{c}{ Range } & & \multicolumn{3}{c}{ Azimuth } \\
\cline { 2 - 3 } & IRW(cells) & PSLR(dB) & ISLR(dB) & IRW(cells) & PSLR(dB) & ISLR(dB) \\
\hline $\mathrm{T} 4$ & 1.5000 & -13.2699 & -9.9237 & & 1.1875 & -13.2544 & -9.7706 \\
\hline $\mathrm{T} 7$ & 1.5000 & -13.2342 & -9.8749 & & 1.1875 & -13.1715 & -9.5636 \\
\hline
\end{tabular}




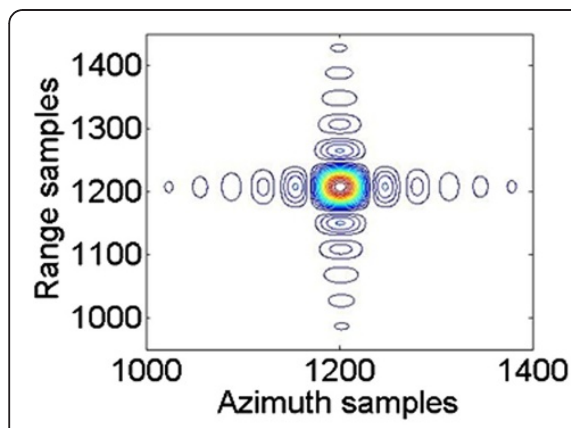

(a)

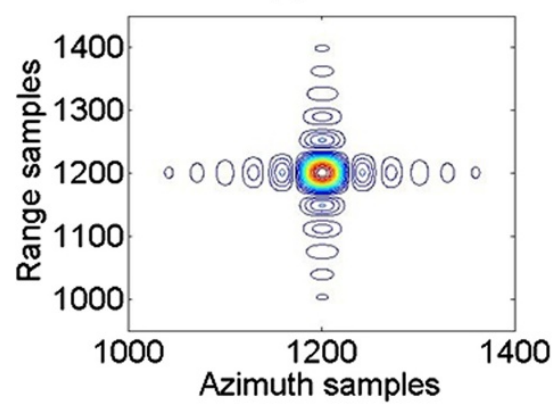

(d)

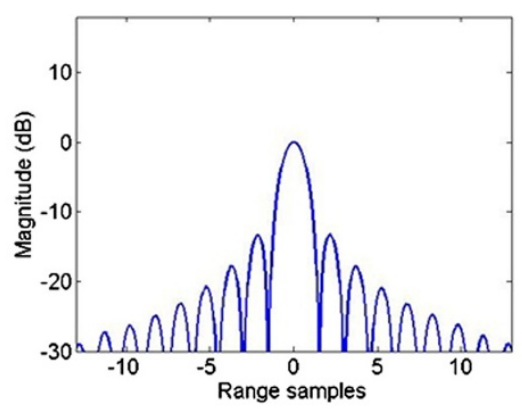

(b)

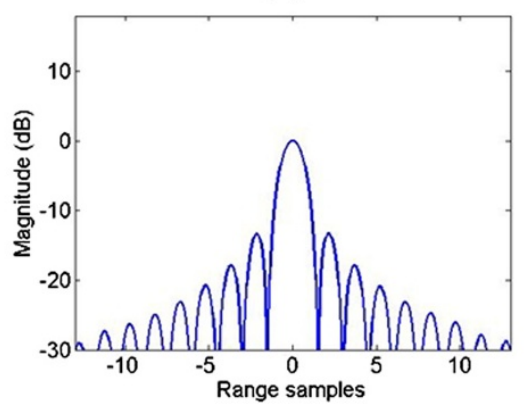

(e)

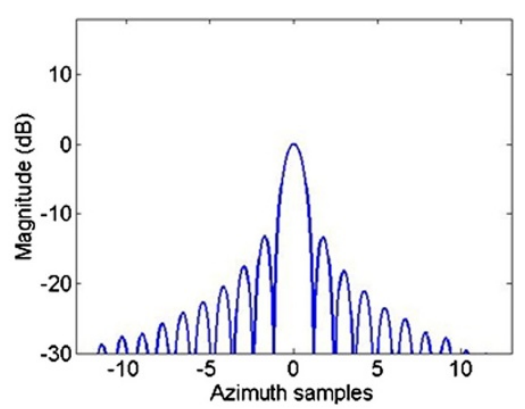

(c)

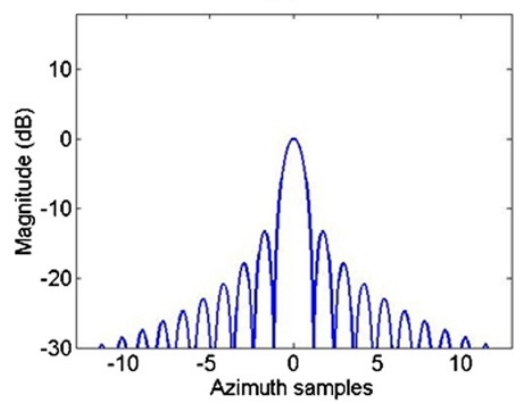

(f)

Figure 5 Imaging results of target T4 for Case II: (a) contour plots obtained by using the proposed chirp-scaling algorithm; (b) range impulse response obtained by using the proposed chirp-scaling algorithm; (c) azimuth impulse response obtained by using the proposed chirp-scaling algorithm; (d) contour plots obtained by using the MSR spectrum-based algorithm; (e) range impulse response obtained by using the MSR spectrum-based algorithm; ( $f$ ) azimuth impulse response by using the MSR spectrum-based algorithm.

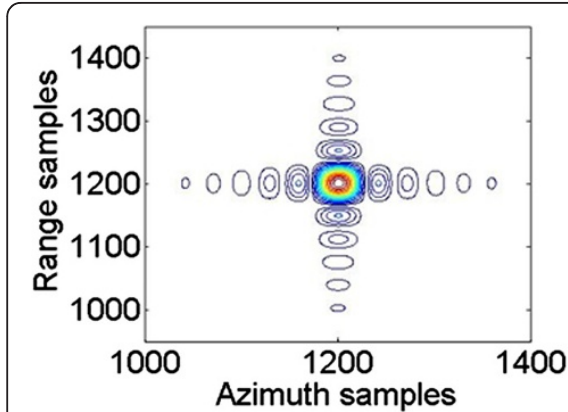

(a)

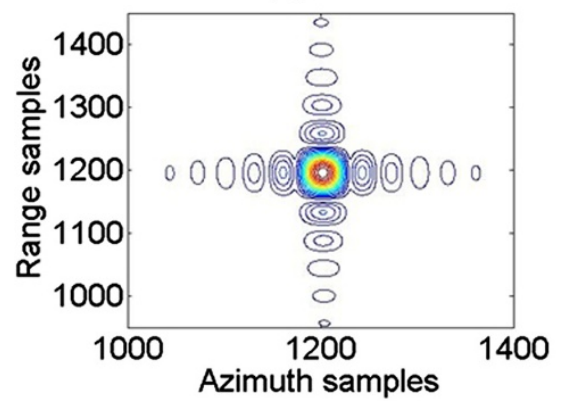

(d)

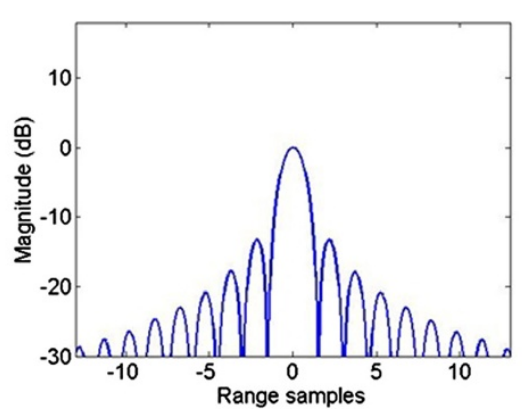

(b)

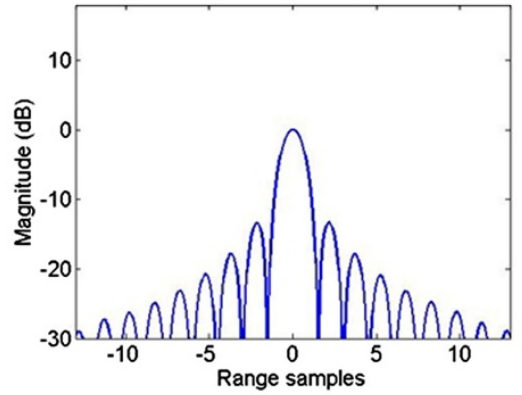

(e)

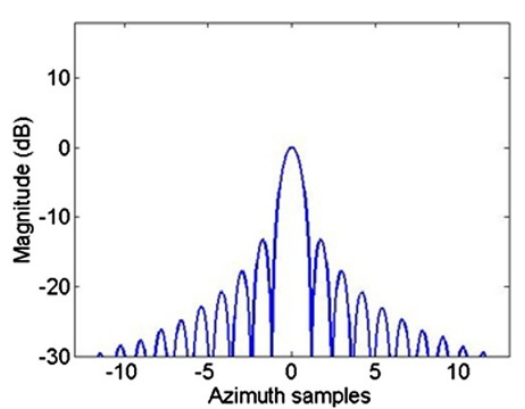

(c)

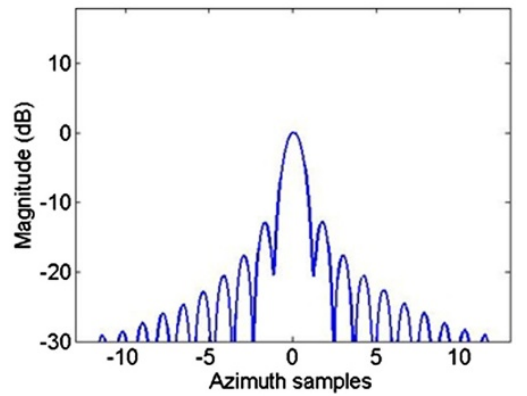

(f)

Figure 6 Imaging results of target T5 for Case II: (a) contour plots obtained by using the proposed chirp-scaling algorithm; (b) range impulse response obtained by using the proposed chirp-scaling algorithm; (c) azimuth impulse response obtained by using the proposed chirp-scaling algorithm; (d) contour plots obtained by using the MSR spectrum-based algorithm; (e) range impulse response obtained by using the MSR spectrum-based algorithm; (f) azimuth impulse response by using the MSR spectrum-based algorithm. 


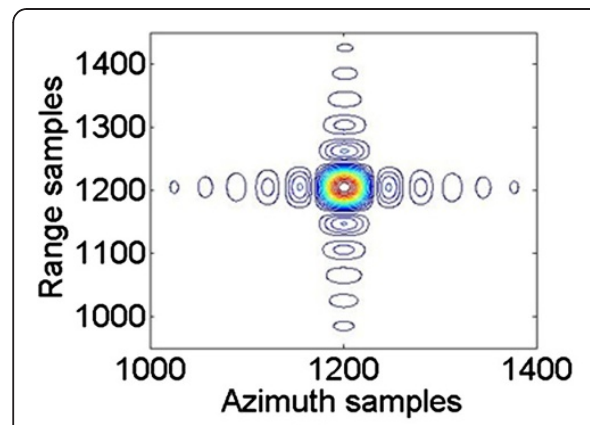

(a)

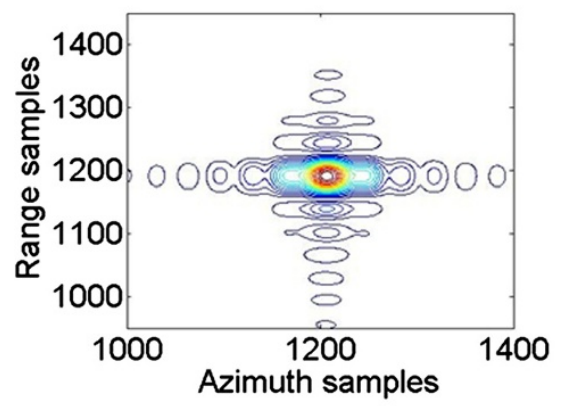

(d)

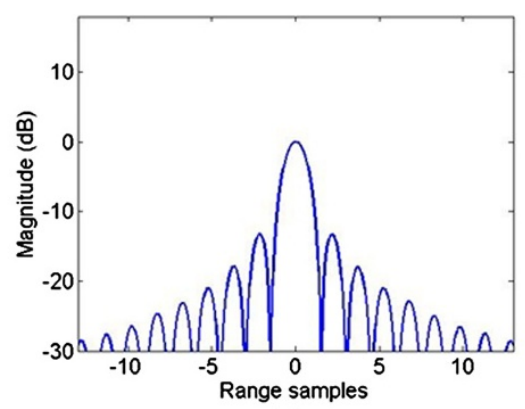

(b)

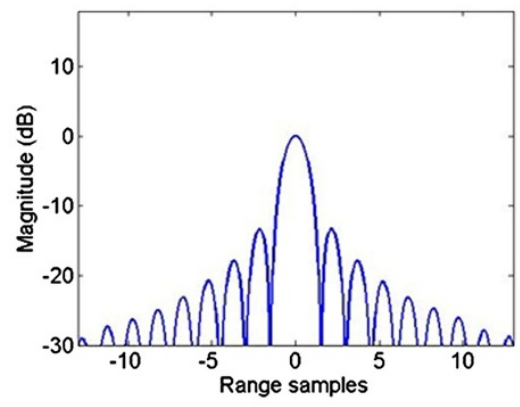

(e)

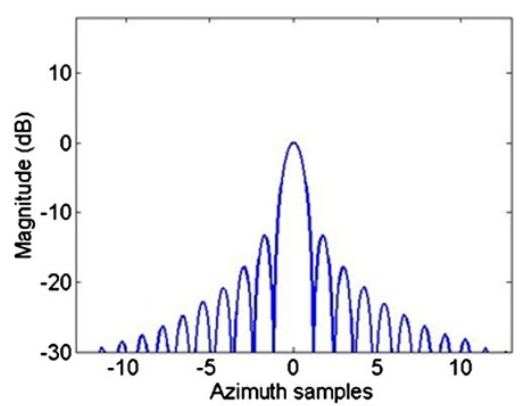

(c)

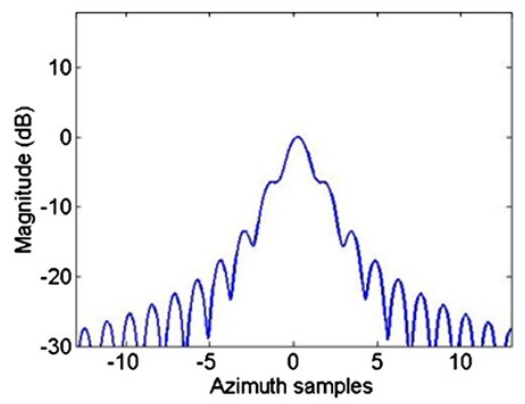

(f)

Figure 7 Imaging results of target T6 for Case II: (a) contour plots obtained by using the proposed chirp-scaling algorithm; (b) range impulse response obtained by using the proposed chirp-scaling algorithm; (c) azimuth impulse response obtained by using the proposed chirp-scaling algorithm; (d) contour plots obtained by using the MSR spectrum-based algorithm; (e) range impulse response obtained by using the MSR spectrum-based algorithm; ( $f$ ) azimuth impulse response by using the MSR spectrum-based algorithm.

receiver, the proposed algorithm has better focusing results with large baselines than the one presented in [18] and less computational burden than the BFFBP algorithm. We compare the performance of the algorithms in three situations, we first take a look at their performance with the length of the baseline $6000 \mathrm{~m}$, the slant ranges from the reference target to the transmitter and receiver are both $20224 \mathrm{~m}$, and the corresponding squint angles are $8.53^{\circ}$ and $-8.53^{\circ}$, respectively. The imaging results for the reference point under the three algorithms are shown in Figure 9. As can be seen, satisfying result can be obtained by using all the algorithms. In the following, we come to the situation under the length of the baseline $12,000 \mathrm{~m}$, the slant range is $20,881 \mathrm{~m}$, the squint angles are $16.7^{\circ}$ and $-16.7^{\circ}$ for the transmitter and the receiver, respectively, and corresponding results are displayed in Figure 10. Figure 11 shows the imaging results with the length of the baseline $13,000 \mathrm{~m}$ by using the three algorithms, the slant range is $21,030 \mathrm{~m}$ and the squint angles are $18.0^{\circ}$ and $-18.0^{\circ}$, respectively. From the imaging results, we can tell that, ideal focusing qualities can be obtained by using the proposed algorithm and the BFFBP algorithm for all the cases, while the performance based on the spectrum that is presented in [18] differs much under different situations. All the focusing qualities agree with the theoretical values except for the two results that are obtained by using the chirp-scaling algorithm presented in [18] with the baseline 12,000 and $13,000 \mathrm{~m}$. Corresponding values of the IRW, PSLR, and ISLR for the very two are given in Table 6.

We can see that, with the increasing length of the baseline, the focusing quality will deteriorate by using the algorithm presented in [18]. Since with the increasing length of the baseline in tandem configuration, the precision of the spectrum presented in [18] degrades. Here, we give a brief discussion about this. Neglecting the terms that do not give an influence to the precision of the spectrum, we directly repeat the bistatic PT reference spectrum (BPTRS) that is suitable for general bistatic SAR configuration in [18], which is expressed as $\Psi_{B}\left(f, f_{\tau}\right)=2 \pi R_{0 R} \sqrt{\left(f+f_{0}\right)^{2}-\left(c f_{\tau R} / v_{R}\right)^{2}} / c+2 \pi R_{0 T}$ $\sqrt{\left(f+f_{0}\right)^{2}-\left(c f_{\tau R} / v_{T}\right)^{2}} / c$, where $f$ and $f_{\tau}$ represent the range and Doppler frequencies, $R_{0 R}$ and $R_{0 T}$ are the closest distances from the flight track to the target of the transmitter and the receiver. $v_{T}$ and $v_{R}$ are the velocities of the transmitter and the receiver, respectively. $f_{0}$ is the center frequency. Under the situation of the tandem 


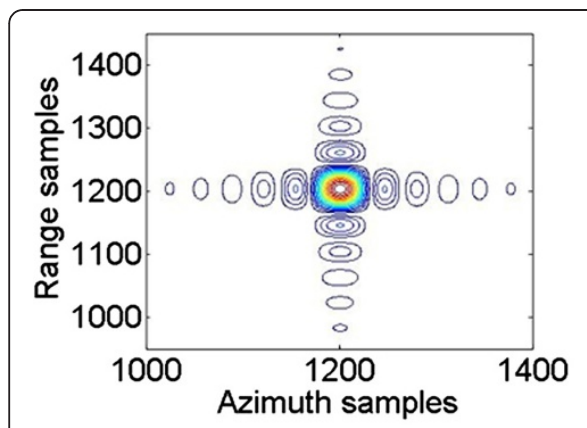

(a)

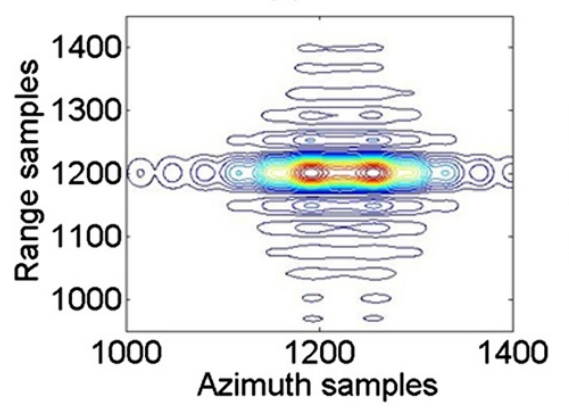

(d)

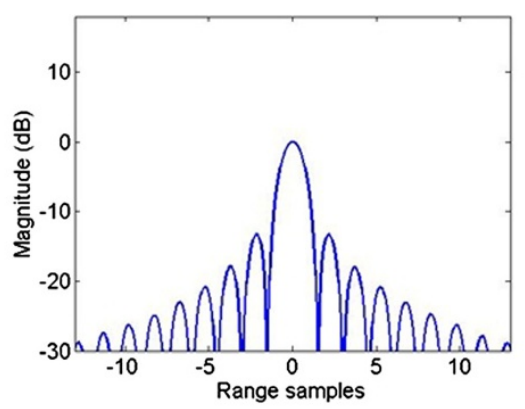

(b)

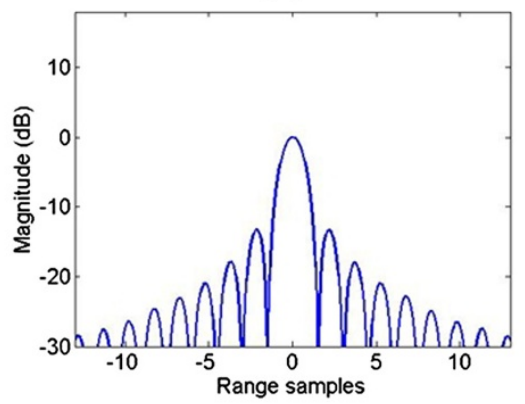

(e)

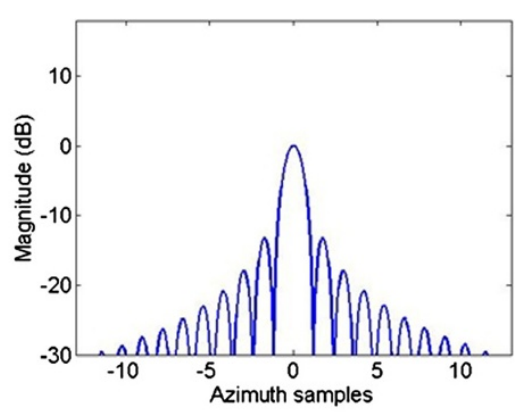

(c)

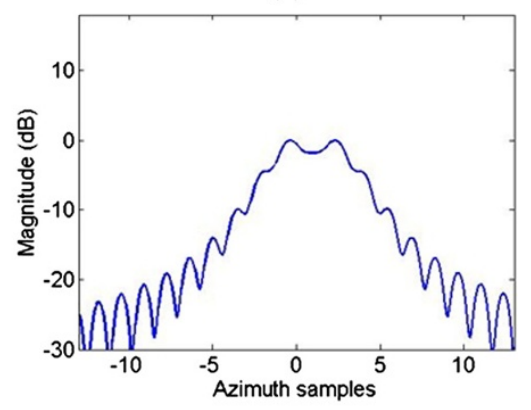

(f)

Figure 8 Imaging results of target T7 for Case II: (a) contour plots obtained by using the proposed chirp-scaling algorithm; (b) range impulse response obtained by using the proposed chirp-scaling algorithm; (c) azimuth impulse response obtained by using the proposed chirp-scaling algorithm; (d) contour plots obtained by using the MSR spectrum-based algorithm; (e) range impulse response obtained by using the MSR spectrum-based algorithm; ( $f$ ) azimuth impulse response by using the MSR spectrum-based algorithm.

configuration discussed here, the expression will degenerate into a much more simple formation as $\Psi_{B}\left(f, f_{\tau}\right)=$ $4 \pi R_{B} \sqrt{\left(f+f_{0}\right)^{2}-\left(c f_{\tau} / 2 v\right)^{2}} / c$. Then, we transform it into the wavenumber domain $\Psi_{B}\left(K_{R}, K_{X}\right)=$ $R_{B} \sqrt{4 K_{R}^{2}-K_{X}^{2}}$. To see the relationships of the spectrum and the exact analytical one that we are based on in this article, we divide the exact analytical PT spectrum into two parts $\Psi_{1}=R_{B} \sqrt{4 K_{R}^{2} \cos ^{2} \beta-K_{X}^{2}}$ and $\Psi_{2}\left(K_{R}, K_{X}\right)=$ $h_{x} \tan \beta \sqrt{4 K_{R}^{2} \cos ^{2} \beta-K_{X}^{2}}$. We can see that both spectrums will become the familiar spectrum of the monostatic case when $h_{x}=0, \beta=0$. And from the

Table 4 Quality parameters of impulse-response function for targets T4-T7 in Case II by using the proposed algorithm

\begin{tabular}{lcccccccc}
\hline & \multicolumn{3}{c}{ Range } & & \multicolumn{3}{c}{ Azimuth } \\
\cline { 2 - 3 } & IRW(cells) & PSLR(dB) & ISLR(dB) & IRW(cells) & PSLR(dB) & ISLR(dB) \\
\hline $\mathrm{T} 4$ & 1.5000 & -13.2785 & -9.9150 & & 1.1875 & -13.2871 & -9.7493 \\
\hline $\mathrm{T} 5$ & 1.5000 & -13.2719 & -9.8815 & & 1.1875 & -13.2708 & -9.7440 \\
\hline $\mathrm{T} 6$ & 1.5000 & -13.2622 & -9.8408 & & 1.1875 & -13.2631 & -9.7101 \\
\hline $\mathrm{T} 7$ & 1.5000 & -13.2394 & -9.7629 & & 1.1875 & -13.2327 & -9.6975 \\
\hline
\end{tabular}

comparison between $\Psi_{B}$ and $\Psi_{1}$, we can see that the difference between them is the factor $\cos \beta$, when $\cos \beta$ is very close to 1 , the precision of these two terms are very close, however, $\Psi_{2}$ has been ignored in this case of the BPTRS in [18]. And the error between these two spectrums can be described as $\left|\left(R_{B}+h_{x} \tan \beta\right) \sqrt{4 K_{R}^{2} \cos ^{2} \beta-K_{X}^{2}}-R_{B} \sqrt{4 K_{R}^{2}-K_{X}^{2}}\right|$. From the expression, we can tell that, with the increasing length of the baseline, the error will get larger.

As to the BFFBP algorithm [7], satisfactory imaging results can be obtained without the influence of the length of the baseline. Here, we compare its computational burden with the proposed frequency domain imaging

Table 5 Quality parameters of impulse-response function for targets T4-T7 in Case II by using the MSR-based algorithm

\begin{tabular}{|c|c|c|c|c|c|c|}
\hline & \multicolumn{3}{|c|}{ Range } & \multicolumn{3}{|c|}{ Azimuth } \\
\hline & IRW(cells) & PSLR(dB) & ISLR(dB) & IRW(cells) & PSLR(dB) & $\operatorname{ISLR}(\mathrm{dB})$ \\
\hline T4 & 1.5000 & -13.2781 & -9.7334 & 1.1875 & -13.2737 & -9.7544 \\
\hline T5 & 1.5000 & -13.2642 & -9.6772 & 1.2500 & -12.7284 & -9.2906 \\
\hline$\overline{\mathrm{T} 6}$ & 1.5000 & -13.2419 & -9.5948 & 1.5625 & -6.4846 & -4.5136 \\
\hline T7 & 1.5000 & -13.2251 & -9.5552 & 4.6875 & -0.0054 & -1.7584 \\
\hline
\end{tabular}




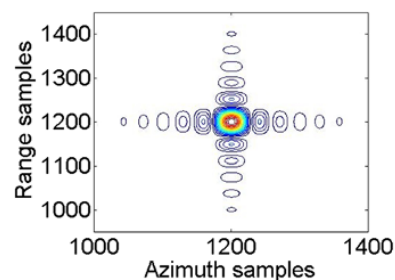

(a)

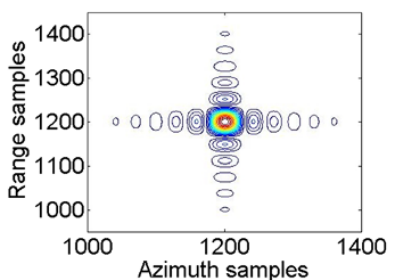

(b)

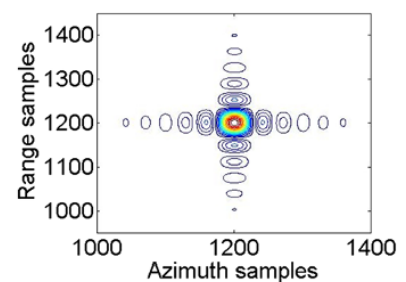

(c)

Figure 9 Contour plots of with the length of the baseline 6,000 m: (a) by using the chirp-scaling algorithm presented in [18]; (b) by using the BFFBP algorithm; (c) by using the proposed chirp-scaling algorithm.

algorithm. We estimate the number of floating point operations (FLOPs) for the proposed algorithm and the BFFBP algorithm, each FLOP can either be a real multiply or a real add [24].

As is known, an FFT operation of length $N_{0}$ requires $5 N_{0} \cdot \log _{2}\left(N_{0}\right)$ FLOPs, and a complex phase multiply requires six FLOPs [24]. First, we estimate the computational burden of the proposed bistatic chirp-scaling algorithm with $N_{r g}$ range samples and $N_{a z}$ azimuth samples, which consists of the following main parts as discussed in the previous section: FFTs in azimuth, multiplication by the chirp-scaling function, FFTs in range, phase multiplication, IFFTs in range, phase multiplication in azimuth, and IFFTs in azimuth. And each step requires $5 N_{r g} \cdot N_{a z} \cdot \log _{2}\left(N_{a z}\right), 6 N_{r g} \cdot N_{a z}, 5 N_{a z} \cdot N_{r g}$. $\log _{2}\left(N_{r g}\right), 6 N_{r g} \cdot N_{a z}, 5 N_{a z} \cdot N_{r g} \cdot \log _{2}\left(N_{r g}\right), 6 N_{r g} \cdot N_{a z}$, and $5 N_{r g} \cdot N_{a z} \cdot \log _{2}\left(N_{a z}\right)$ FLOPs, respectively.

As for the BFFBP algorithm, the computational burden mainly consists of the computational burden of the back-projection part of the DBP process and the computational burden of the interpolation of two grids into a new one in the elliptical coordinate throughout the stages of the algorithm. To compare the computational burden with the proposed frequency domain imaging algorithm, we estimate the FLOPs required by each processing step, we define $\mu_{\mathrm{DBP}}$ the number of FLOPs that is needed for each back-projection in the range direction, while $\mu_{\mathrm{INT}}$ the number of FLOPs that is needed for the interpolation of two grids into a new one of the elliptical coordinate throughout the stages of the BFFBP algorithm. And according to [7] where the split factor is 2, $N_{a z}=2^{N}$ and $K$ is the number of the splits of the aperture, we can tell that the total FLOPs needed for the back-projection part of the DBP process are proportional to $u_{\mathrm{DBP}} \cdot N_{r g} \cdot N_{a z} \cdot 2^{N-K}$, and the FLOPs needed for the interpolation of each stage are $u_{\mathrm{INT}} \cdot N_{r g} \cdot N_{a z} \cdot 2$. And the total FLOPs needed for the whole process of the BFFBP algorithm will be $u_{\mathrm{DBP}} \cdot N_{r g} \cdot N_{a z} \cdot 2^{N-K}+u_{I N T} \cdot N_{r g} \cdot N_{a z} \cdot 2 \cdot K$.

For bistatic case, each back-projection needs to calculate the distance from the chosen grid to the transmitter and the receiver, respectively [7]. In tandem configuration, for any grid in the elliptical coordinate, we only have to calculate the distance from the grid to the transmitter to get the slant range sums due to the simple geometry formation. And the total computational burden of each back-projection for tandem configuration requires about $\mu_{\mathrm{DBP}}=100$ FLOPs, which mainly consists of the calculation of the distance from the chosen grid to the transmitter, the calculation of the slant range sums, range interpolation, and phase multiply, where an eight-point sinc interpolator is used for range

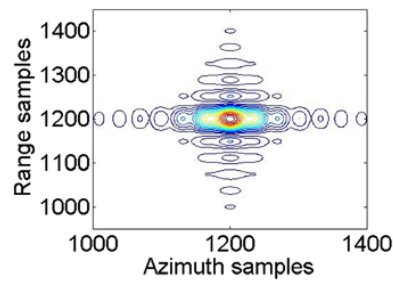

(a)

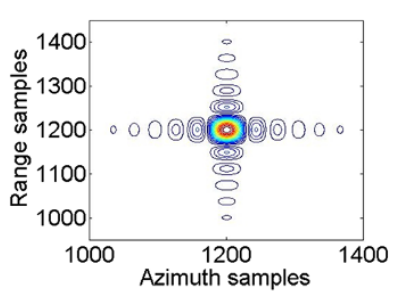

(b)

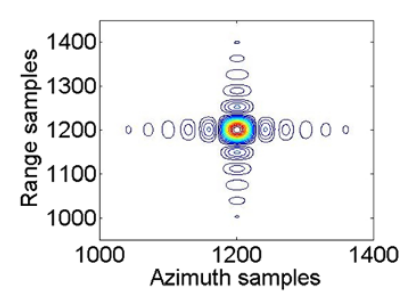

(c)

Figure 10 Contour plots of with the length of the baseline 12,000 m: (a) by using the chirp-scaling algorithm presented in [18]; (b) by using the BFFBP algorithm; (c) by using the proposed chirp-scaling algorithm. 


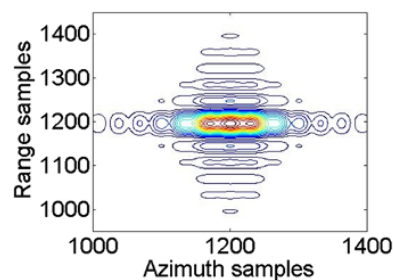

(a)

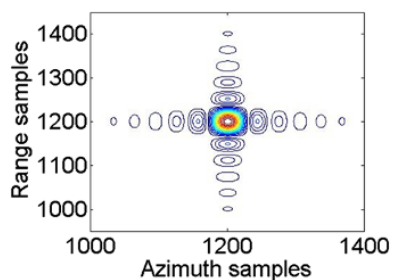

(b)

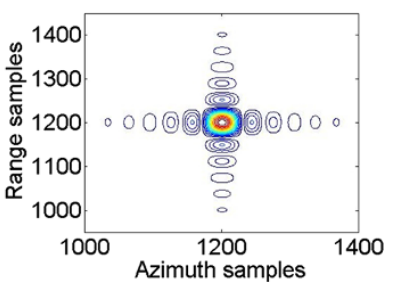

(c)

Figure 11 Contour plots of with the length of the baseline $13,000 \mathrm{~m}$ : (a) by using the chirp-scaling algorithm presented in [18]; (b) by using the BFFBP algorithm; (c) by using the proposed chirp-scaling algorithm.

interpolation, and a conservative estimation of $\mu_{\mathrm{INT}}$ will be about 640 FLOPs if a 2D eight-point sinc interpolator is used for range and angle interpolation [7]. Hereto, we can give a rough estimation of the FLOPs needed for the whole implementation of the BFFBP algorithm, the FLOPs required by the BFFBP algorithm for tandem configuration will be $100 \cdot N_{r g} \cdot N_{a z} \cdot 2^{N-K}+640 \cdot N_{r g} \cdot N_{a z} \cdot 2 \cdot K$. (Here, we only give a rough estimation of the FLOPs that is needed for the processing of the BFFBP algorithm, and the computational burden may be different in different bistatic SAR configurations. Besides, the computational burden of the interpolation is different in different situations. The better the required precision is, the more computational burden of the interpolation will be. The quality of the image is a trade-off between the accuracy and the computational burden [7].)

To see the comparison more clearly, we give an illustration of the computational burden of these two algorithms. We take $N_{\text {ref }}=N_{r g}=N_{a z}=2048$ for example. The FLOPs required of the proposed algorithm are $5 \cdot N_{\text {ref }}^{2}$. $\log { }_{2}\left(N_{\text {ref }}\right)+6 \cdot N_{\text {ref }}^{2}+5 \cdot N_{\text {ref }}^{2} \cdot \log _{2}\left(N_{\text {ref }}\right)+6 \cdot N_{\text {ref }}^{2}+5 \cdot N_{\text {ref }}^{2} \cdot$ $\log _{2}\left(N_{\text {ref }}\right)+6 \cdot N_{\text {ref }}^{2}+5 \cdot N_{\text {ref }}^{2} \cdot \log _{2}\left(N_{\text {ref }}\right)=0.9982 \times$ $10^{9}$ FLOPs. And as to the BFFBP algorithm, in accordance with [7], where the split factor is chosen to be 2, and the number of splits is chosen by $K=N-1, N=\log _{2} N_{\text {ref }}=11$ and a $2 \mathrm{D}$ eight-point sinc interpolator is used for range and angle interpolation. The total FLOPs required of the BFFBP algorithm are $100 \cdot N_{r g} \cdot N_{a z} \cdot 2^{N-K}+640 \cdot N_{r g} \cdot N_{a z}$. $2 \cdot K=54.5260 \times 10^{9}$ FLOPs. From the results, we can see that the proposed algorithm has an advantage over the BFFBP algorithm in the view of computational burden. Moreover, the efficiency can be improved greatly using GPU for the BFFBP algorithm [25].

From these two comparisons, we can draw the conclusion that although the algorithms presented in [18] and the BFFBP algorithm [7] can be applied into more general configurations and therefore have wider application areas. Focusing on the tandem configuration, the proposed algorithm can achieve satisfying imaging results with small computational burden.

\section{Conclusion}

An exact analytical PT spectrum-based chirp-scaling algorithm is presented to correct the RCM for tandem bistatic SAR data processing. Unlike the existing algorithms, it is no longer sensitive to the baseline to range ratio, even when the baseline is equal to the range. Only phase multiplication and FFT are needed during the whole imaging process, which improves the effectiveness of the proposed algorithm. When the bistatic SAR data are obtained with a large baseline in tandem configuration, we believe that the proposed algorithm could be a good choice.

Moreover, motion errors of bistatic SAR are more complex respect to the monostatic case due to its complex formation [26]. And the proposed imaging algorithm is discussed without the consideration of motion errors, the baseline is assumed to be constant during all the acquisition, which is hard to achieve in real bistatic SAR systems. The motion compensation methods are well worth working on.

Table 6 Quality parameters of impulse-response function with the baseline 12,000 and $13,000 \mathrm{~m}$ for the chirp-scaling algorithm presented in [18]

\begin{tabular}{lccccccc}
\hline & \multicolumn{3}{c}{ Range } & & \multicolumn{3}{c}{ Azimuth } \\
\cline { 2 - 8 } & IRW(cells) & PSLR(dB) & ISLR(dB) & & IRW(cells) & PSLR(dB) & ISLR(dB) \\
\hline Baseline $12,000 \mathrm{~m}$ & 1.5000 & -13.2794 & -9.7190 & 1.9375 & -3.9490 & -1.2212 \\
\hline Baseline $13,000 \mathrm{~m}$ & 1.5000 & -13.2609 & -9.7082 & 4.3750 & -0.7295 & 2.5553 \\
\hline
\end{tabular}




\section{Competing interests}

The authors declare that they have no competing interests.

\section{Acknowledgments}

We would like to thank Prof Hongxian Wang and Dr Lei Yang for their patient support during the revision of the manuscript. This study was supported by the National Natural Science Foundation of China under Grants no. 60890072, 61222108 and the "973" Program of China under grants no. 2010 CB731903

Received: 28 June 2012 Accepted: 12 February 2013 Published: 1 March 2013

\section{References}

1. Rodriguez-Cassola, M, Baumgartner, V, Krieger, G, Nottensteiner, A, Krieger, G, \& Moreira, A (2010). Bistatic TerraSAR-X/F-SAR spaceborne-airborne SAR experiment: description, data processing, and results. IEEE Trans. Geosci. Remote Sens., 48(2), 781-794.

2. Walterscheid, I, Espeter, T, Brenner, A, Klare, J, Ender, J, Nies, H, et al. (2010). Bistatic SAR experiments with PAMIR and TerraSAR-X - setup, processing, and image results. IEEE Trans. Geosci. Remote Sens., 48(8), 3268-3279.

3. Rodriguez-Cassola, M, Prats, P, Schulze, D, Tous-Ramon, N, Steinbrecher, U, Marotti, L, et al. (2012). First bistatic spaceborne SAR experiments with TanDEM-X. IEEE Geosci. Remote Sens. Lett., 9(1), 33-37.

4. Weiß, M.(2004). Time and frequency synchronization aspects for Bistatic SAR systems. In Proceedings of EUSAR (pp. 395-398). Ulm, Germany:

5. Krieger, G, \& Moreira, A (2006). Spaceborne bi- and multistatic SAR: potentia and challenges. IET Proc. Radar Sonar Navigat., 153(3), 184-198.

6. Barber, B (1985). Theory of digital imaging from orbit synthetic aperture radar. Int. J. Remote Sens., 6(6), 1009-1057.

7. Rodriguez-Cassola, M, Prats, P, Krieger, G, \& Moreira, A (2011). Efficient timedomain image formation with precise topography accommodation for general bistatic SAR configurations. IEEE Trans. Aerospace Electron. Syst., 47(4), 2949-2966.

8. D'Aria, D, Guarnieri, A, \& Rocca, F (2004). Focusing bistatic synthetic aperture radar using dip move out. IEEE Trans. Geosci. Remote Sens., 42(7), 1362-1376.

9. Ding, J, Zhang, Z, Xing, M, \& Bao, Z (2008). A new look at the bistatic-to -monostatic conversion for tandem SAR image formation. IEEE Geosci. Remote Sens. Lett., 5(3), 392-395.

10. Loffeld, O, Nies, H, Peters, V, \& Knedlik, S (2004). Models and useful relations for bistatic SAR processing. IEEE Trans. Geosci. Remote Sens., 42(10), 2031-2038.

11. Wang, R, Loffeld, O, Neo, Y, Nies, H, \& Dai, Z (2010). Extending Loffeld's bistatic formula for the general bistatic SAR configuration. IET Proc. Radar Sonar Navigat., 4(1), 74-84.

12. Chen, S, Wu, Q, Zhou, P, Xing, M, \& Bao, Z (2012). A new look at the Loffeld's bistatic formula in tandem configuration. IEEE Geosci. Remote Sens. Lett., 9(1), 710-714.

13. Neo, Y, Wong, F, \& Cumming, I (2007). A two-dimensional spectrum for bistatic SAR processing using series reversion. IEEE Geosci. Remote Sens. Lett., 4(1), 93-96.

14. Neo, Y, Wong, F, \& Cumming, I (2008). A comparison of point target spectrum derived for bistatic SAR processing. IEEE Trans. Geosci. Remote Sens., 46(9), 2481-2492.

15. Neo, Y, \& Wong, FH (2008). ICumming, Focusing bistatic SAR data using nonlinear chirp scaling algorithm. IEEE Trans. Geosci. Remote Sens., 46(9), 2493-2505.

16. Liu, B, Wang, T, Wu, Q, \& Bao, Z (2009). Bistatic SAR data focusing using an Omega-K algorithm based on method of series reversion. IEEE Trans. Geosci. Remote Sens., 47(8), 2899-2912.

17. Clemente, C, \& Soraghan, J (2012). Approximation of the bistatic slant range using Chebyshev polynomials. IEEE Geosci. Remote Sens. Lett., 9(4), 682-686.

18. Wang, R, Deng, Y, Loffeld, O, Nies, H, Walterscheid, I, Espeter, T, et al. (2011). Processing the azimuth-variant bistatic SAR data by using monostatic imaging algorithms based on two-dimensional principle of stationary phase. IEEE Trans. Geosci. Remote Sens., 49(10), 3504-3520.

19. Zhang, Z, Xing, M, Ding, J, \& Bao, Z (2007). Focusing parallel bistatic SAR data using the analytic transfer function in the wavenumber domain. IEEE Trans. Geosci. Remote Sens., 45(11), 3633-3645.
20. Wu, Q, Liang, Y, Xing, M, Qiu, C, Bao, Z, \& Yeo, T (2011). Focusing of tandem bistatic-configuration data with range migration algorithm. IEEE Geosci. Remote Sens. Lett., 8(1), 88-92.

21. Wu, Q, Xing, M, Shi, H, Qiu, C, \& Bao, Z (2011). Exact analytical twodimensional spectrum for bistatic synthetic aperture radar in tandem configuration. IET Proc. Radar Sonar Navigat., 5(3), 349-360.

22. Raney, R, Runge, H, Bamler, R, Cumming, I, \& Wong, F (1994). Precision SAR processing using chirp scaling. IEEE Geosci. Remote Sens., 32(4), 786-799.

23. Davidson, G, Cumming, I, \& Ito, M (1996). A chirp scaling approach for processing squint model SAR data. IEEE Trans. Aerospace Electron. Syst., $32(1), 122-133$.

24. Cumming, I, \& Wong, F (). Digital Processing of Synthetic Aperture Radar Data. Algorithms and Implementation. ArtechHouse, Norwood, MA, 2005:

25. Ponce, O, Prats, P, Rodriguez-Cassola, M, Scheiber, R, \& Reigber, A (2011). Processing of circular SAR trajactories with fast factorized back-projection. In Proceedings of IGARSS (pp. 3692-3695). Vancouver Canada:

26. Rigling, B, \& Moses, R (2006). Motion measurement errors and autofocus in bistatic SAR. IEEE Trans. Imag. Process., 15(4), 1008-1016.

doi:10.1186/1687-6180-2013-38

Cite this article as: Chen et al.: Focusing of tandem bistatic SAR data using the chirp-scaling algorithm. EURASIP Journal on Advances in Signal Processing 2013 2013:38.

\section{Submit your manuscript to a SpringerOpen ${ }^{\circ}$ journal and benefit from:}

- Convenient online submission

- Rigorous peer review

- Immediate publication on acceptance

- Open access: articles freely available online

- High visibility within the field

- Retaining the copyright to your article

Submit your next manuscript at springeropen.com 